\begin{tabular}{|c|l|}
\hline Title & Identification of silicate and carbonaceous presolar grains by SIMS in the type 3 enstatite chondrite A LHA 81189 \\
\hline Author(s) & Ebata, Shingo; Fagan, Timothy J.; Y urimoto, Hisay oshi \\
\hline Citation & $\begin{array}{l}\text { A pplied Surface Science, 255(4), 1468-1471 } \\
\text { https://doi.org/10.1016/.apsusc.2008.05.048 }\end{array}$ \\
\hline Issue Date & 2008_12-15 \\
\hline Doc URL & http://hdl.handle.net/2115/38755 \\
\hline Type & article (author version) \\
\hline File Information & 255-4_p1468_1471.pdf \\
\hline
\end{tabular}

Instructions for use 


\title{
Identification of Silicate and Carbonaceous Presolar Grains by SIMS in the Type 3 Enstatite Chondrite ALHA81189
}

\author{
Shingo Ebata ${ }^{\mathrm{a}^{*}}$, Timothy J. Fagan ${ }^{\mathrm{b}}$ and Hisayoshi Yurimoto ${ }^{\mathrm{a}}$ \\ ${ }^{a}$ Department of Natural History Sciences, Hokkaido University, Sapporo 060-0810, Japan. \\ ${ }^{\mathrm{b}}$ Department of Earth Sciences, School of Education, Waseda University, Tokyo, Japan.
}

\begin{abstract}
An isotope ratio imaging technique using the HokuDai isotope microscope system has been applied to in-situ survey for presolar grains in the type-3 enstatite chondrite ALHA81189. Rastered and static ion beam were used for primary beam. Lateral resolution of the isotope image was achieved to be $0.4 \mu \mathrm{m}$ for static ion beam mode and to be $0.6 \mu \mathrm{m}$ for rastered ion beam mode. As a result, the abundances of presolar grains are 150 to $200 \%$ larger under the static ion beam mode than under the rastered ion beam mode. Development of image processing introducing isotopography of ${ }^{32} \mathrm{~S}^{-},{ }^{24} \mathrm{Mg}^{16} \mathrm{O}^{-}$and ${ }^{56} \mathrm{Fe}^{-}$succeeded to increase efficiency of presolar grain characterization. Using the static ion beam and introducing appropriate isotopography were very useful methods of in-situ characterization of presolar grains in meteorites.
\end{abstract}

Keywords: Isotope microscope; SIMS; Imaging; Isotope ratio; Presolar grain; Meteorite

\section{Introduction}

Primitive meteorites contain presolar grains that predate the formation of our solar system. We know about stellar nucleosynthesis and the evolution from isotopic compositions of the presolar grains. Carbonaceous and oxide grains have been identified as a type of presolar grains and recently silicate grains have been found by O-isotope ion imaging with a NanoSIMS [1, 2], with isotope microscope (Cameca ims$1270+$ SCAPS [3]) [e.g. 4].

Although about 200 presolar silicates were discovered, the chemical compositions of only 27 presolar silicate grains have been studied in meteorites [e.g. 2,4], IDPs [e.g. 1] and AMMs [e.g. 5], because it is difficult to specify presolar silicates embedded in abundant solar system silicates that are major constituents of meteorites. The spatial resolution and sensitivity of the conventional ion imaging technique have been difficult to identify sub-micron presolar silicate grains because of the small sizes (average sizes of identified grains $\sim 300$ $\mathrm{nm}$ ). Among 27 presolar silicate grains, eight have compositions similar to olivine, eleven are pyroxene-like, and seven have GEMS (glass with embedded metal and sulfides)-like compositions. The sizes of presolar silicates seems to be between 100 and $1000 \mathrm{~nm}$ with most grains less than 300 nm.

Ebata et al. [6] have discovered presolar silicate and carbonaceous grains from three type 3 enstatite chondrites by in-situ measurements. However, grain characterizations were limited to $\sim 17 \%$ of all presolar grains found. Developments of the effective characterization method are required. 
Isotope microscope is a mass spectrometer that has capability of permil-precision isotope ratio imaging with micro-scale resolution under high mass-resolution [3]. This instrument is achieved by the combination of a stigmatic SIMS instrument and a two-dimensional ion detector SCAPS (stacked CMOS-type active pixel sensor). The stigmatic SIMS can project the isotope distribution of the sample surface to the SCAPS with keeping the positional information. The isotope image obtained using this method is called "isotopography" [4].

Here we report the developments of characterization for silicate and carbonaceous presolar grains in the type 3 enstatite chondrite ALHA81189 using isotopography.

\section{Experimental}

\subsection{Presolar grain survey}

We surveyed presolar grains by isotopography using a Hokudai isotope microscope system (Cameca ims 1270 and SCAPS). In this study we compared rastered and static primary ion irradiation for improvement of spatial resolution of secondary ion images.

Under the rastered ion beam condition, a $20-\mathrm{keV} \mathrm{Cs}{ }^{+}$primary ion beam of $5 \mu \mathrm{m}$ in diameter was rastered over a $80 \times 80 \mu \mathrm{m}^{2}$ sample area to obtain homogeneous secondary ion-beam emission from an imaging area of $75 \times 75 \mu \mathrm{m}^{2}$. On the other hand, under the static beam condition, a 20$\mathrm{keV} \mathrm{Cs}{ }^{+}$primary ion beam with $75 \mu \mathrm{m}$ in diameter was used to obtain secondary ionbeam emission. The shaped beam illumination mode was applied to obtain homogenous secondary ion-beam emission from the illumination area. A normal incident electron gun was used for charge compensation of the rastered area. The primary current was adjusted to $\sim 0.5 \mathrm{nA}$ for both mode. In order to obtain better lateral resolution of isotopography than the case of [4], a smaller contrast aperture $(50 \mu \mathrm{m}$ in diameter) was used in this study except for the case of $\mathrm{C}$-isotopes. A larger contrast aperture (150 $\mu \mathrm{m}$ in diameter) was used for $\mathrm{C}$-isotopography in order to minimize sample consumption by sputtering with an enough precision of isotope ratio through the sequence. Lateral resolutions of the isotopography using contrast aperture of the $50 \mu \mathrm{m}$ and of the $150 \mu \mathrm{m}$ are $0.3-0.5 \mu \mathrm{m}$ and $\sim 1 \mu \mathrm{m}$, respectively. These analytical conditions keep the sputtering depth less than $100 \mathrm{~nm}$ for the sequence.

Under the rastered ion beam condition, we acquired the following isotopography for each analyzing field as a sequence of ${ }^{12} \mathrm{C}^{-}$, ${ }^{13} \mathrm{C}^{-},{ }^{12} \mathrm{C}^{-},{ }^{27} \mathrm{Al}^{-},{ }^{28} \mathrm{Si}^{-},{ }^{16} \mathrm{O}^{-},{ }^{18} \mathrm{O}^{-},{ }^{16} \mathrm{O}^{-},{ }^{17} \mathrm{O}^{-}$, and ${ }^{16} \mathrm{O}^{-}$. Total integration time for one field was $\sim 1$ hour.

Under the static beam condition, we acquired the following isotopography for each analyzing field as a sequence of ${ }^{12} \mathrm{C}^{-}$, ${ }^{13} \mathrm{C}^{-},{ }^{12} \mathrm{C}^{-},{ }^{27} \mathrm{Al}^{-},{ }^{28} \mathrm{Si}^{-},{ }^{16} \mathrm{O}^{-},{ }^{18} \mathrm{O}^{-},{ }^{16} \mathrm{O}^{-},{ }^{17} \mathrm{O}^{-}$, ${ }^{16} \mathrm{O}^{-},{ }^{16} \mathrm{OH}^{-},{ }^{32} \mathrm{~S}^{-},{ }^{24} \mathrm{Mg}^{16} \mathrm{O}^{-}$and ${ }^{56} \mathrm{Fe}^{-}$. Total integration time for one field was $\sim 1.5$ hour because the number of the isotopography is larger in this mode.

The digital image processing, the selection criterion for distinguishing presolar grains and estimation of the analytical errors of the grains are the same as [4].

\subsection{Presolar grain characterization}

For positioning presolar grains in a back scattered electron image, we carefully compared among all elemental images by the isotope microscope and high resolution back scattered electron images by scanning electron microscope. After positioning the isotopically anomalous spot area on a backscattered electron image, we applied quantitative microanalysis to determine the 
chemical composition of the isotopically anomalous spot. The Mineralogical and petrographical characterization of isotopic anomalous grains and surrounding matrix has been conducted using a field emission type scanning electron microscope (JEOL JSM-7000F) equipped with energy dispersive X-ray spectrometer (Oxford INCA Energy).

Elemental maps were collected with 5 and $15 \mathrm{kV}$ of accelerating voltage for determining pinpoint the exact location of this grain. The $5 \mathrm{kV}$ irradiation condition is more suitable for collecting light elements, especially carbon.

Quantitative microanalyses were performed with 5 and $15 \mathrm{kV}$ electron probe with the beam current of 3.0 and $0.9 \mathrm{nA}$, respectively. The estimated spatial resolution is 0.3 and $1 \mu \mathrm{m}$ for 5 and $15 \mathrm{kV}$ electron probe condition, respectively.

\section{Results and discussion}

\subsection{Abundances and Lateral resolution}

The lateral resolution under rastered ion beam mode was $\sim 0.6 \mu \mathrm{m}$, whereas lateral resolution under static ion beam mode, was
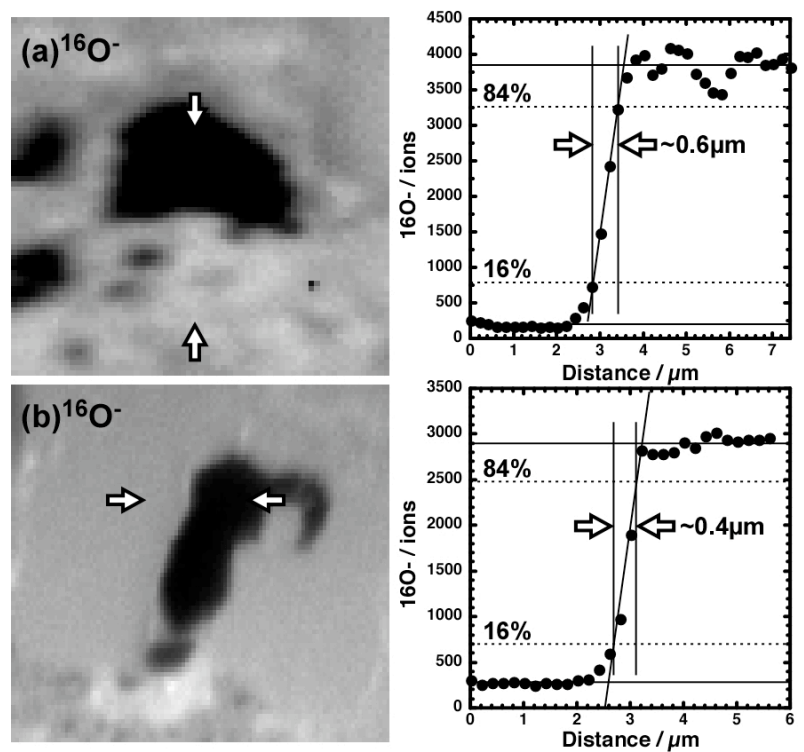

Fig. 1. Lateral resolution of isotopography. (a) Lateral resolution was $\sim 0.6 \mu \mathrm{m}$ using rasterized ion beam. (b) Lateral resolution was $\sim 0.4 \mu \mathrm{m}$ using static ion beam. $\sim 0.4 \mu \mathrm{m}$ (Fig. 1). These lateral resolutions were improved more the five times comparing with previous work [6]. The lateral resolution is more improved under the static ion beam mode. This indicates that the static ion radiation introduces stable electrostatic condition of sample surface comparing with the rastered ion irradiation probably because the secondary ion images are sensitive to the variation of the electrostatic field on the sample surface.

Volume abundances of presolar silicate and carbonaceous grains in matrix were obtained to be $\sim 14$ and $\sim 9 \mathrm{ppm}$, respectively, assuming the mean grain size to be $0.3 \mu \mathrm{m}$ in diameter, respectively, under the rastered ion beam mode, whereas were obtained to be $\sim 22$ and $\sim 17 \mathrm{ppm}$, respectively, under the static ion beam mode.

The abundances of presolar silicate and carbonaceous grains under the static ion beam mode increases of $\sim 60$ and $\sim 90 \%$ larger than those under the rastered ion beam mode, respectively [7].

Silicate/carbonaceous-grain ratio under static ion beam mode $(\sim 1.3 \pm 0.3)$ is almost the same as those under rastered ion beam

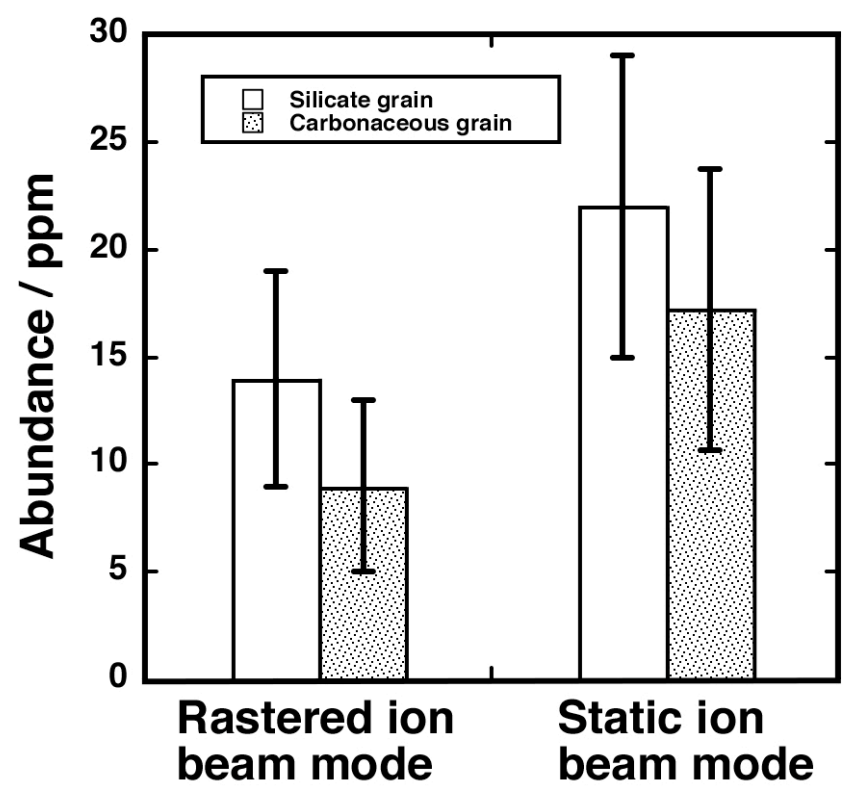

Fig. 2. The abundances of presolar silicate and carbonaceous grains in the case of using rastered and static ion beam. Error bars are $2 \sigma$. Silicate/C-grain ratios are calculated to be $\sim 1.6$ for rastered ion beam mode and $\sim 1.3$ for static ion beam mode. 
mode $(\sim 1.6 \pm 0.4)$ (Fig.2). This indicates that the increase of presolar grain abundances under the static ion beam mode is not due to the heterogeneous distribution between the two types of presolar grain in the meteorite. The abundance increase is consistent to the improvement of spatial resolution of isotopography.

\subsection{Characterization of presolar grains}

Characterization efficiency for presolar grains has been limited to $\sim 11 \%$ for silicate grains and $0 \%$ for carbonaceous grains [6]. New isotopographies of ${ }^{32} \mathrm{~S}^{-},{ }^{24} \mathrm{Mg}^{16} \mathrm{O}^{-}$and ${ }^{56} \mathrm{Fe}^{-}$were very useful of grain characterization (Fig. 3). The elements of $\mathrm{Mg}, \mathrm{S}$, and $\mathrm{Fe}$ were major phase forming elements in the meteorites and are useful to position matching between isotopography and the corresponding images of electron microscope. Therefore it makes easier to determine the presolar grain position. Especially, $\mathrm{Mg}$ isotope image is very useful because we can distinguish between olivine (indicated by white arrow grains in Fig.3a) and pyroxene (gray grains in Fig.3a, which
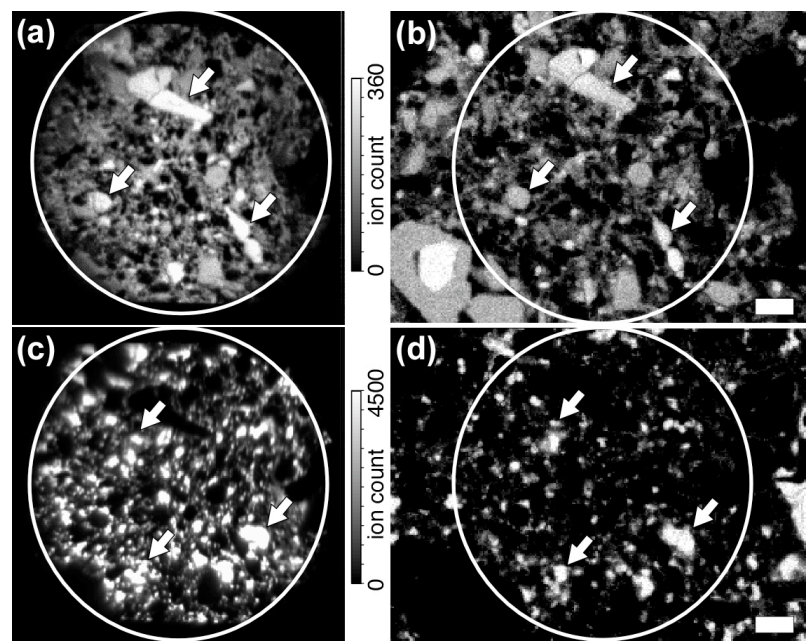

Fig. 3. Comparison between secondary ion image by isotope microscope and the elemental map by scanning electron microscope. (a) ${ }^{24} \mathrm{Mg}^{16} \mathrm{O}^{-}$ isotopography (b) $\mathrm{Mg}$ map (c) ${ }^{32} \mathrm{~S}^{-}$isotopography (d) $\mathrm{S}$ map. White circles show the equivalent areas for (a) and (b) and for (c) and (d), respectively. White arrows indicate characteristic grains in the images of (a) and (b) and of (c) and (d), respectively. are not indicated by arrows).

Six presolar silicate grains were determined the chemical compositions [8]. Three presolar carbonaceous grains were determined the chemical compositions [8]. Grain characterization efficiency increases to $60 \%$ for silicate presolar grains and $\sim 43 \%$ for carbonaceous presolar grains.

Introduction of isotopography of ${ }^{32} \mathrm{~S}^{-}$, ${ }^{24} \mathrm{Mg}^{16} \mathrm{O}^{-}$and ${ }^{56} \mathrm{Fe}^{-}$was succeeded to develop the presolar grain characterization in the meteorite.

\section{Conclusions}

In-situ presolar grains survey was performed with isotope microscope. Grain characterization efficiency increased to $60 \%$ for silicate and $\sim 43 \%$ for carbonaceous using static ion beam and ${ }^{32} \mathrm{~S}^{-},{ }^{24} \mathrm{Mg}^{16} \mathrm{O}^{-}$and ${ }^{56} \mathrm{Fe}^{-}$isotopography. These results indicate that it is very useful for characterization of presolar grains to use static ion beam for improving lateral resolution and ${ }^{32} \mathrm{~S}^{-}$, ${ }^{24} \mathrm{Mg}^{16} \mathrm{O}^{-}$and ${ }^{56} \mathrm{Fe}^{-}$isotopography as major position markers.

\section{Acknowledgements}

We thank T. McCoy and L. Welzenbach for loaning us the thin section of ALHA81189. We also thank S. Kobayashi, N. Sakamoto and $\mathrm{S}$. Itoh for the many useful discussions and their help with solving numerous technical problems.

\section{References}

[1] Messenger et al. (2003) Science 300, 105-108.

[2] Nguyen and Zinner (2004) Science 303, 14961499.

[3] Yurimoto et al. (2003) Appl. Surf. Sci. 203-204, 793.

[4] Nagashima et al. (2004) Nature 428, 921-924.

[5] Yada et al. (2006) LPS XXXVII, 1470.

[6] Kunihiro et al. (2005) GCA 69, 763-773.

[7] Ebata et al. (2006) LPS XXXVII, 1619.

[8] Ebata et al. (2007) MaPS 40, A38, 5150. 